\title{
The Optics Suitcase: educational outreach tool for inspiring careers in light
}

Jessica DeGroote Nelson, Tanya Kosc, Phillip Nelson

Jessica DeGroote Nelson, Tanya Z. Kosc, Phillip C. Nelson, "The Optics Suitcase: educational outreach tool for inspiring careers in light," Proc. SPIE 11143, Fifteenth Conference on Education and Training in Optics and

Photonics: ETOP 2019, 111432N (2 July 2019); doi: 10.1117/12.2523798

SPIE Event: Fifteenth Conference on Education and Training in Optics and Photonics: ETOP 2019, 2019, Quebec City, Quebec, Canada 


\title{
The Optics Suitcase: Educational Outreach Tool for Inspiring Careers in Light
}

\author{
Jessica DeGroote Nelson*a,d ${ }^{\text {, Tanya Z. Kosc }}{ }^{\text {b,d }}$, Phillip C. Nelson ${ }^{c}$ \\ aOptimax Systems, Inc., 6367 Dean Parkway, Ontario NY 14519; 'baboratory for Laser Energetics, \\ University of Rochester, 250 East River Road, Rochester NY 14623, 'Masline Electronics, 511 \\ South Clinton Ave, Rochester NY 14620; 'OSA Rochester Section, www.osarochester.org; \\ *jnelson@optimaxsi.com; phone 1585 265-1020;
}

\begin{abstract}
Developed by the OSA Rochester Section (OSA-RS), the Optics Suitcase is an innovative, interactive presentation package designed to introduce middle school students to the dynamic and exciting range of concepts within the study of light. The Optics Suitcase contains supplies and giveaway theme packets for in-class presentations that explore color in white light. The goal is to help promote technology careers to middle school age students. A detailed presentation guide is included with the suitcase to help give presenters techniques for engaging students during the presentation and making the demonstrations more interactive. Three experiments explore the colors constituting white light in the form of diffraction (The Rainbow Peephole), polarization (Magic Stripes) and selective reflection (Magic Patch). These three experiments use giveaway theme packets that are designed to help reinforce the study of light concepts at home as students present the information they learned to their family and friends at home. This paper outlines the contents of the Optics Suitcase, a typical lesson plan as well as tips for giving an Optics Suitcase presentation.
\end{abstract}

Keywords: Educational Outreach, Optics Suitcase, Lesson Plan

\section{INTRODUCTION AND OBJECTIVE}

The Optics Suitcase (see Figure 1) is an educational outreach tool developed by Dr. Stephen D. Jacobs and the OSA Rochester Section (OSA-RS) with the busy professional in mind. It is designed to make it easy to enter a middle school classroom and excite young people about careers in technology using experiments that can be customized to highlight the presenter's interests, job and work environment. The Optics Suitcase contains both reusable and giveaway supplies (see Table 1).

The objective of the Optics Suitcase is to convey a sense of excitement about technology in a short period of time. To achieve this goal, the initial demonstrations serve as "ice breakers" and are intended to quickly capture the students" attention. Next, three hands-on activities use the theme packets and illustrate the overall theme of "colors in white light". They are presented as a pace best suited (as determined by the presenter) to retain the students' interest, and children often enjoy taking the theme packets home and sharing with others. The presenter can customizing the template take-home flyer with a name, the date and the location of the presentation to help reinforce the message with the students.

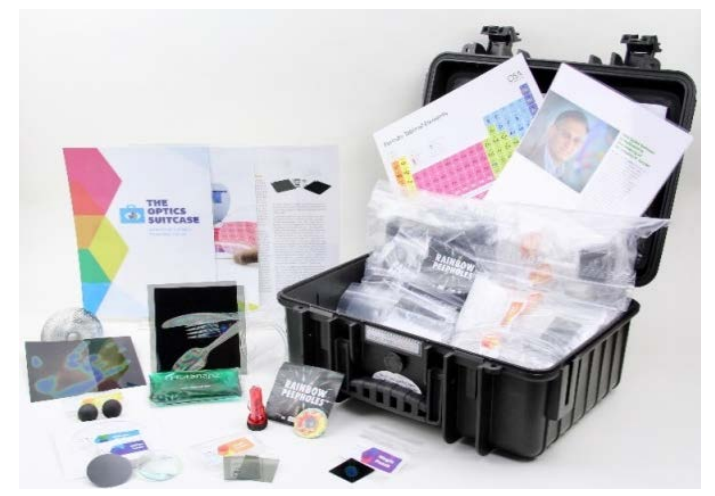

Figure 1: Photograph of the Optics Suitcase and its contents.

Fifteenth Conference on Education and Training in Optics and Photonics: ETOP 2019, edited by

Anne-Sophie Poulin-Girard, Joseph A. Shaw, Proc. of SPIE Vol. 11143, 111432N · C 2019

SPIE, ICO, IEEE, OSA · CCC code: 0277-786X/19/\$18 · doi: 10.1117/12.2523798 


\begin{tabular}{|cl|}
\hline Quantity & Item - Reusable Supplies \\
\hline $\mathbf{1}$ & Durable suitcase with room for other items that can be added to customize \\
\hline $\mathbf{1}$ & Instruction guide on laminated sheets \\
\hline $\mathbf{1}$ & USB-stick with supplemental Optics Suitcase materials \\
\hline $\mathbf{1}$ & Hot Snapz - heat pad \\
\hline $\mathbf{1}$ & Set of Arbor Scientific “Happy and Unhappy” balls \\
\hline $\mathbf{1}$ & Slinky \\
\hline $\mathbf{1}$ & 50 mm Silicon wafer, one side polished to a “mirror” finish \\
\hline $\mathbf{1}$ & Silica glass lens \\
\hline $\mathbf{2}$ & 5” x 5” pieces of high-quality sheet polarizer \\
\hline $\mathbf{4}$ & Transparent plastic cups \\
\hline $\mathbf{1}$ & Set of transparent plastic tableware: fork, spoon, knife \\
\hline $\mathbf{1}$ & 6” x 6” sheet of temperature-sensitive microencapsulated liquid crystal sheet \\
\hline Quantity & Item - Giveaway Supplies (can be re-supplied) \\
\hline $\mathbf{5 0}$ & Rainbow Peephole: Color by Diffraction \\
\hline $\mathbf{5 0}$ & Magic Stripes: Color by Polarized Transmission \\
\hline $\mathbf{5 0}$ & Magic Patch: Color by Selective Reflection \\
\hline $\mathbf{5 0}$ & Periodic Table of Elements \\
\hline & Table 1: List of Optics Suitcase reusable and giveaway supplies \\
\hline
\end{tabular}

\section{PREPARING FOR A VISIT TO THE CLASSROOM}

One to two weeks before the visit, it is recommended to obtain the following information from the classroom teacher to prepare the suitcase and make a take-home flyer:

- School name, school address, teacher's name, classroom location

- $\quad$ Ages of students

- Number of students

o Take four extra sets of theme packets and flyers (one for the teacher, one for you, and two spares)

- Length of presentation

- Number of presentations

o Will you speak to one or two classes?

- The setting and teacher's expectations

o Is your visit part of a career day at school where others will be describing their occupations as well?

o Is your visit being used to emphasize experiments that demonstrate fun with science?

o Is your visit being used as part of a science nit on the topic of physics?

- $\quad$ Protocol for entering the school

o You will probably report to the school office for a badge.

- Appropriate arrival time

o 20 minutes before the target class time?

o Will there be time available to setup in the classroom?

- Would the teacher like a short biography to introduce you to the class?

- Are there any restrictions on taking pictures?

The day before the visit, the presenter should check the contents of the suitcase. It is necessary to ensure that the heating demo pad is in its liquid phase (see Section 3), to print out the flyers (included a digital copy that can be projected as part of the presentation, and to find time to practice the presentation. 


\section{GETTING STARTED (BREAKING THE ICE)}

Presentation times are often limited to a class period ( $\sim 5$ minutes). It is very important to grab the students' attention immediately and to maintain a quick pace for the entire presentation. After the teacher or one of the students has completed the introduction, start with two general science demos to help break the ice.

\subsection{Heating Pad Demo}

Pick a student from the class and ask him/her to help with the first experiment. Hold up the heat pad without indicating what it is and hand it to the student. Ask him/her to gently handle the heat pad and describe how it looks and feels (squishy, transparent, liquid, and cool to the touch). The student may need to be gently prompted to get these answers. Ask the student to hold up the heating pad so that everyone can see it, allowing the metal disk inside the pad to sink to the bottom of the pad. Help the student to snap the metal disk to initiate crystallization. Describe how the crystallization, accompanied by heat, spreads throughout the pad in approximately 30 seconds. The pad turns opaque. Prompt the child to describe the pad, now that it has become hard and hot! Pass the heating pad around, so that everyone can feel the heat, while providing additional information:

- This demo is an example of an exothermic reaction in a super-saturated solution

- This commercial product is an instant heating pad for therapy and treatment of certain aches and pains.

- Training in chemistry and similar technologies provided someone with the tools to invent this product.

- The heating pad is reusable. Place the heating pad into boiling water for several minutes to dissolve the crystals.

Put the heating pad back into the Optics Suitcase.

\subsection{Happy and Unhappy Balls}

Select two students to help with the next experiment. Give each student a rubber ball and ask them if the balls look and feel the same. (The answer is "yes".) Ask each student to bounce his/her ball in a location where others can see the zone of contact between the ball and hard surface (desktop, wall or floor). One ball will bounce; the other will not. Explain that this demonstration is an example of polymer and materials science.

- The "Happy Ball" is fully vulcanized; its composition was engineered to resist being deformed by contact with a hard surface. It will roll down an incline at a faster speed than the "Unhappy Ball", since its coefficient of friction is lower.

- The "Unhappy Ball” deforms to absorb the shock of contact with a hard surface. It has no bounce.

Questions for the students might be: What is a good rubber for the bumper on a car? What rubber would be best for a handball, or the soles of a pair of sneakers? This is a good time to point out that technology and innovation are the keys to improving our lives. Put the demo can then be put back into the Suitcase.

\section{INTRODUCTION TO OPTICAL ENGINEERING}

Once the students' attention has been captured, it is time to introduce the field of technology called "Optical Engineering”. Hold up the silicon wafer (shiny side out) and the silica glass lens (see Figure 2). Ask if anyone can identify these "optics". The lens is usually easily recognized and described. The students will likely refer to the wafer as a mirror. Identify the wafer as single-crystal silicon, a pure elemental substance and the basis for all computers (the chips and microcircuits). Show the students the reverse side of the wafer, which is dull, and explain that this side is ground and the other is polished to a mirror surface. Some optical engineers develop the technologies for turning rough silicon wafers into integrated circuits for making computer chips. You may want to elaborate on this. 


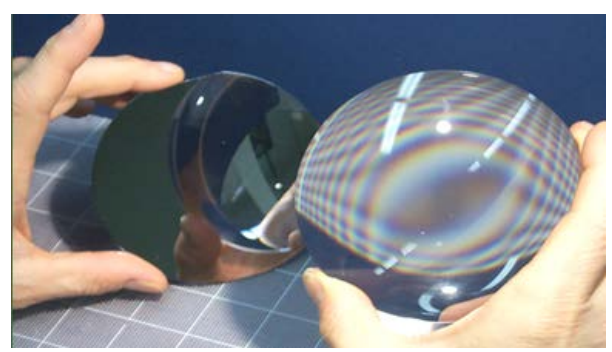

Figure 2: Photograph of a silicon wafer and silica lens

Hand out copies of the periodic table of elements (useful for 8-9th grade and higher). State that the periodic table is a visual means for displaying all of the elements known to man - every bit of matter in the universe is composed of one or more of these elements. Ceramics engineers, chemists, materials scientists, geologists, and optical engineers work with many of these elements and the compounds they form. Explain that the only difference between the silicon wafer and the silica lens is oxygen. Help the students locate silicon (\#14) and oxygen (\#8). Point out that the addition of oxygen turns a visibly opaque material into a visibly clear one. Suggest that if we were aliens whose vision was in the infrared, the silica lens would look opaque and the silicon wafer would look transparent! Note that many insects have infrared vision. Mention that optical engineers build lenses into systems that image light, such as the Hubble Space Telescope, the Chandra X-ray Telescope, digital cameras and smart phones.

\section{COLORS IN WHITE LIGHT}

Once the students are engaged and have had a brief introduction to Optical Engineering, conduct three experiments that illustrate optical engineering. These experiments explore several ways to "see" the colors in white light. The demos can be described as revealing "secrets" that might not be obvious to others when doing the experiments. The "trick" or "secret" aspect to the demos often works well to continue to engage students. This is a good time to project an image of the takehome flyer (see Figure 3) that describes optics and what it takes to be successful in this career. They flyer can be passed out at this time or at the end of the presentation as a summary. The three experiments that the students will perform are: the Rainbow Peephole (color by diffraction), the Magic Stripes (color by polarized transmission) and the Magic Patch (color by selective reflection).

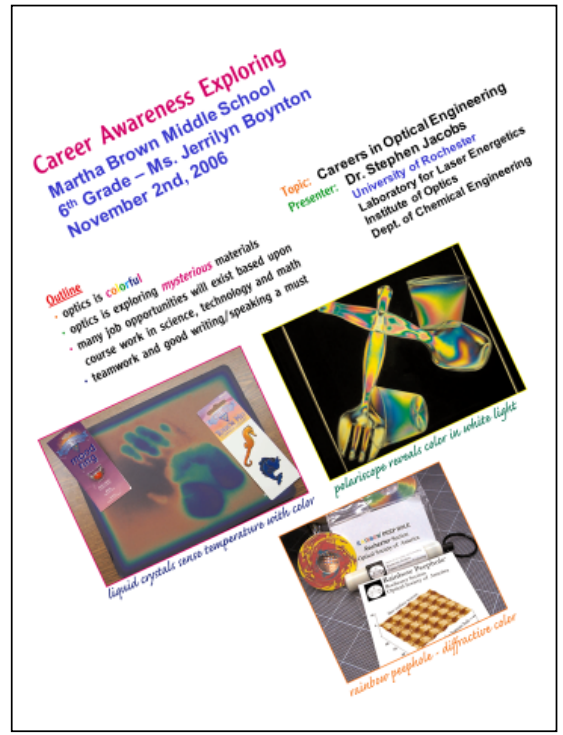

Figure 3: Example take-home flyer 


\subsection{Rainbow Peephole (Color by Diffraction)}

Distribute the Rainbow Peephole theme packets (see Figure 4a), but ask the students to not open them yet. When everyone has their own packet, remove all of the items from the packet, hold them up and identify them. Direct the students to remove the contents from their packet. Hold the peephole up to your eye and look at the flashlight through it. Ask the students to do the same. During the "oohs" and "ahs," ask the group, "Where does the color come from?" Many children will answer that the colors come from the peephole. Tell them that the color comes from the white light in the flashlight. There are several follow-up questions that can be asked. Do you see a regular pattern? Describe it. Identify all of the colors. Are they the same in each spot? Does the pattern change if the flashlight is close or far away? Do you see colors from other people's flashlights, even those far away from you? Do you see colors from the room lights? Are colors and patterns from all light sources the same?

The image on the packet has an atomic force microscope (AFM) image of one side of the clear plastic in the peephole. This is a good opportunity to mention that the AFM is a tool often used by engineers to help see objects at high magnifications. The scale on the image is in microns, which may not be a unit of measure the students are familiar. It can be noted that a human hair is 30 to 80 microns wide, and the bumps across the peephole are only two microns high, which is too small to be seen or felt. The bumps are packed so closely together that about fifty could fit inside a human hair. These bumps are responsible for breaking up the light coming into the peephole. This effect is called "diffraction". It occurs when light travels through small apertures (e.g., holes or slits). Point out the similarity between the regular array of bumps and the pattern seen though the peephole.

A great application example is telecommunications. Ask for a show of hand of everyone that has or wants a cell phone. Using the slide shown in Figure $4 \mathrm{~b}$, explain how telecommunications use fibers and lasers to divide light from one beam into many, each with a different color. It can be explained that this is the key to unlimited number of conversations all over the world at the same time. At this time, ask the students to put the Rainbow Peephole experiment away and tell them that the packets are theirs to keep. Remind them to reveal the secret of seeing colors in white light through diffraction to family members.
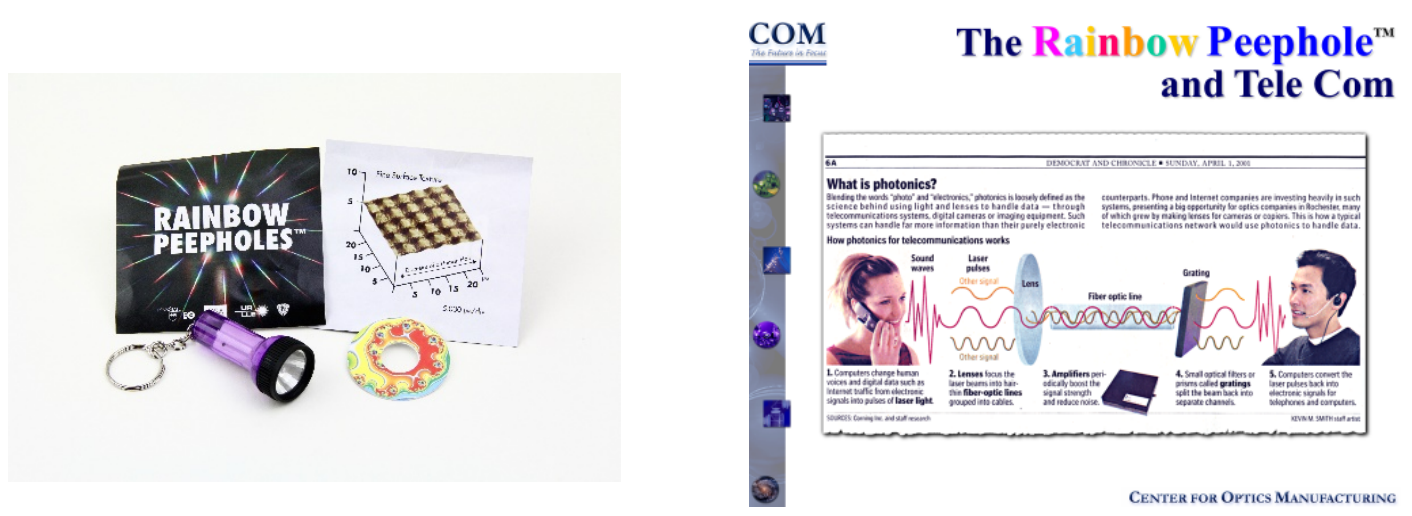

Figure 4: (a) The Rainbow Peephole theme packet and (b) slide on telecommunications application

\subsection{Magic Stripes (Color by polarized transmission)}

To start the next experiment, choose a volunteer to come to the front of the room. Give the student one end of the slinky and ask her/him to hold their end steady and mid-chest level. The presenter should stand approximately four feet away and begin to shake the slinky randomly: up and down and in a circle. The presenter should be able to create a standing wave with a few nodes, but the plane of vibration should not be well defined.

State that, in addition to color, light has a wave nature. The slinky represents a light wave. The motion represents unpolarized light, or light without a preferred vibration direction. For simplicity, it is recommended to ignore circularly polarized light. At this point, stop the circular motion and vibrate only vertically. The presenter should state that light is "polarized" when it vibrates in one direction, vertical or horizontal. While explaining, switch to a horizontal hand motion. The horizontal motion is a bit harder to maintain while speaking, so switch back to vertical motion, if necessary. Define 
linearly polarized light as light that vibrates in a plane. The NASA slide (see Figure 5) showing the spectrum from radio to gamma rays is an additional way to introduce different wavelengths of radiation. The lower photos show how the Milky Way looks when viewed through different telescopes that "see" at various wavelengths of optical radiation.

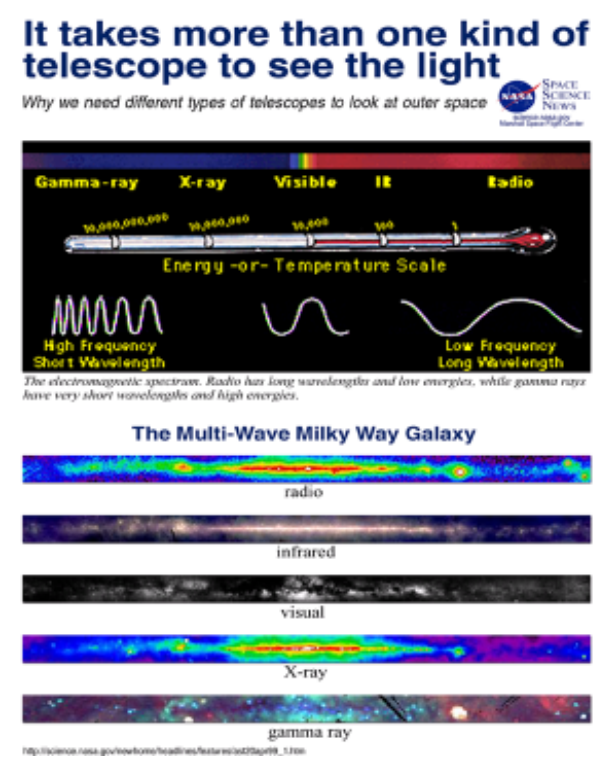

Figure 5: NASA slide showing different wavelengths of light

At this point the Magic Stripes theme packets (see Figure 6a) can be handed out, and again, instruct the students not to open them until everyone has received a packet. Take the two larger pieces of linear sheet polarizer and holds them up, one in each hand. Placing the combined pieces in front of the presenters face allows the students to see the transmission when the axis are parallel and then crossed after rotation. Ask students to remove everything from the packet and place the items on their workspace. Tell the students that the sheets of plastic are called "polarizers" that only allow light vibrating in one direction to pass through and therefore rotating the polarizers in opposite directions prevents any light from passing through. Now they can try to perform the same experiment with their two smaller linear sheet polarizer from their packets. Mention that polarized sunglasses are an example of a commercial application.

If there is an overhead projector in the room, darken the room. Create and project a polariscope by placing one polarizer directly on the light bulb, positioning four plastic cups on the corners of the polarizer, and placing the second polarizer on top of the cups. Place clear plastic silverware included in the Optics Suitcase in the polariscope. Students are typically very excited about what they see. Ask the students where the color comes from. At this point in the presentation, there might be some correct responses. If someone has glasses, ask them to place them in the polariscope. Explain that the stresses inside transparent materials degrade the quality of the linearly polarized light coming through the polariscope and cause various colors to show up. Careers and applications that use polarizers include geologists identifying certain crystals and mineral structures or optics technicians evaluating the quality of laser glasses and laser crystals.

With the room lights back on, show the students how to create their own polariscope with the items in their packet. The polariscope can be held in one hand and the clear plastic items can be placed between the crossed polarizers in the other hand. The students can look through the crossed polarizer "sandwich" at the overhead lights. The slide shown in Figure $6 \mathrm{~b}$ can be projected to show the students the experimental procedure and also to indicate that the colors they are seeing indicate how much stress (optical retardance) is present in the transparent material. The students can apply additional stress on the tongs of the fork and see the effects in their handheld polarizer. As the students are asked to put the Magic Stripes theme packet away, suggest that the students demonstrate the Magic Stripes to trick their families, since they now know the secret polarizer code and how to construct a polariscope. 

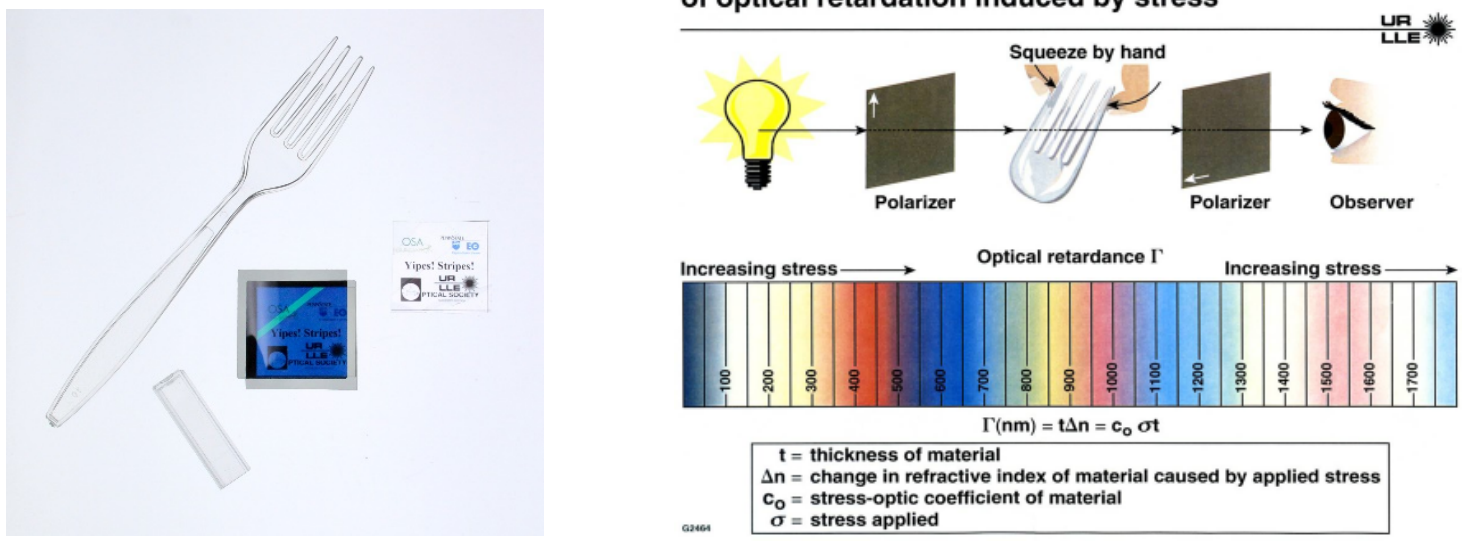

Figure 6: (a) Magic Stripes theme packet and (b) slide to show optical retardation induced by stress

\subsection{Magic Patch (Color by selective reflection)}

Before starting the third experiment, remind the students that he/she has demonstrated how to see colors in white light through diffraction and polarized transmission. While all the students are watching, take the large sheet of microencapsulated liquid crystal and place it in front of a volunteer's face (glasses off, if they wear them), shiny side out. Ask where the colors are coming from. If students guess the heat comes from the presenter's face, respond no. Explain that the colors come from the white room lights reflecting off the black "paper". As the teacher passes out the Magic Patch theme packets (see Figure 7a), explain that the paper has a liquid crystal fluid trapped, or encapsulated, against the shiny side. At the right temperature $\left(84-91^{\circ} \mathrm{F}\right)$, the liquid crystal droplets reflect selective colors of white light. When the liquid crystal sheet is cold there is no reflection (of visible light) and the sheet appears black. When the liquid crystal start to warm, they start to reflect red light. If they warm up sufficiently, they will reflect deep blue light. This effect is called selective reflection. The slide included on the USB stick can be used to compare liquid crystal molecules to springs. Commercial applications that students may be familiar with are "mood rings" or fish tank thermometers. Included on the USB is a slide showing a potential medical application that also gives a subtle message about smoking (see Figure 7b). Once all of the students have their Magic Patch theme packets, instruct them to take their patch out of the bag and perform the vampire test by placing the patch on their wrist. Vampires are the living dead, and give off no heat. The presenter will ask if anyone can "see" a vein. The effect is reversible. As students put the Magic Patches away, remind them that liquid crystals are found in everything from cell phones to watches to laptop computers to video games. It allows an additional opportunity to reinforce the importance of engineers and scientists to develop the technology we all use every day.

A Subtle Message About Smoking

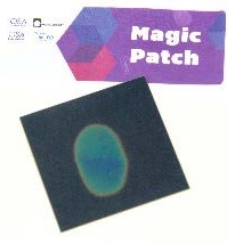

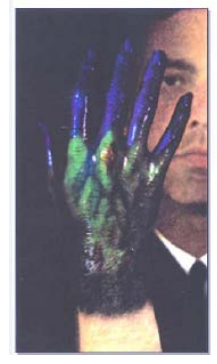

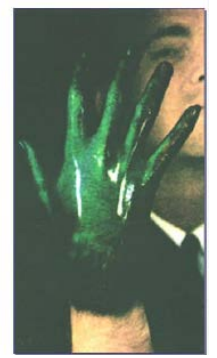

Figure 7: (a) Magic Patch theme packet and (b) slide showing a potential medical application 


\section{SUGGESTIONS AND TIPS}

After giving dozens of presentations, Steve Jacobs and other members of the OSA Rochester Section learned quite a few lessons. A few general observations and suggestions are provided:

- Make arrangements with the teacher ahead of time to have an overhead projector, screen and table at the front of the room. The table will ensure that there is enough room to spread out.

- The presentation is most successful when given to the $6^{\text {th }}-10^{\text {th }}$ grades. Younger children are excited by the gifts, but the message seems to get lost. Note that some of the 'secret' and 'magic' comments should probably be omitted for high schoolers.

- Ask the teacher for help in handing out the theme packets and flyers and for operating the room lights.

- It takes a minimum of 40 minutes to do the presentation as described in the guide. With more time, you can slow the pace, add more personal information, and entertain more questions. With less time, one may want to cover only two activities.

- Adjusting the flow of your presentation is important. Read your audience and move on if their attention seems to be wandering. There is plenty of material.

\section{ACKNOWLEDGEMENTS}

Developed by the OSA Rochester Section (OSA-RS) in 1999, the Optics Suitcase is an innovative, interactive presentation package designed to introduce middle school students to the dynamic and exciting range of concepts within the study of light. To request an Optics Suitcase, please visit us online at www.osarochester.org

Funding for this program comes from many sources. We would like to acknowledge the OSA Rochester Section, the Laboratory for Laser Energetics (LLE) of the University of Rochester, Masline Electronics Inc. and the OSA Foundation for major support.

Dr. Stephen D. Jacobs made lasting contributions to the fields of optical materials, liquid crystals, and optics manufacturing, but it is his passion for educational outreach which continues to impact countless young (and older) lives. He spent his entire career at the University of Rochester with appointments at the Laboratory for Laser Energetics (LLE), Institute of Optics, Materials Science and Chemical Engineering departments. He volunteered as the educational outreach chair for the OSA-Rochester Section for over 15 years during which time he developed and organized the Optics Suitcase program that has been instrumental in introducing hundreds of thousands of young children to the fields of optics and materials science throughout the US and globally.

Steve Jacobs touched many lives.

His vision and desire to share optics continue with the every use of the Optics Suitcase.

\section{REFERENCES}

[1] Reference Optics Suitcase Guide found at website: https://www.osarochester.org/resources/Documents/EducOutreach/OSA_OpticsSuitcaseGuide_020718[19509].pdf 